\title{
Long noncoding RNA GSEC promotes neutrophil inflammatory activation by supporting PFKFB3-involved glycolytic metabolism in sepsis
}

\author{
Dadong Liu ${ }^{1,8}$, Wen Sun ${ }^{2,8}$, Danying Zhang ${ }^{3,8}$, Zongying Yu ${ }^{4}$, Weiting Qin ${ }^{5}$, Yishu Liu ${ }^{6}$, Kai Zhang (D) ${ }^{7 凶}$ and Jiangtao Yin (D) ${ }^{\circledR 凶}$
}

(c) The Author(s) 2021

Metabolic reprogramming is a hallmark of neutrophil activation in sepsis. LncRNAs play important roles in manipulating cell metabolism; however, their specific involvement in neutrophil activation in sepsis remains unclear. Here we found that 11 IncRNAs and 105 mRNAs were differentially expressed in three transcriptome datasets (GSE13904, GSE28750, and GSE64457) of gene expression in blood leukocytes and neutrophils of septic patients and healthy volunteers. After Gene Ontology biological process analysis and IncRNA-mRNA pathway network construction, we noticed that GSEC IncRNA and PFKFB3 were co-expressed and associated with enhanced glycolytic metabolism. Our clinical observations confirmed the expression patterns of GSEC IncRNA and PFKFB3 genes in neutrophils in septic patients. Performing in vitro experiments, we found that the expression of GSEC IncRNA and PFKFB3 was increased when neutrophils were treated with inflammatory stimuli. Knockdown and overexpression experiments showed that GSEC IncRNA was essential for mediating PFKFB3 mRNA expression and stability in neutrophil-like dHL-60 cells. In addition, we found that GSEC IncRNA-induced PFKFB3 expression was essential for mediating dHL-60 cell inflammatory cytokine expression. Performing mechanistic experiments, we found that glycolytic metabolism with PFKFB3 involvement supported inflammatory cytokine expression. In summary, our study uncovers a mechanism by which GSEC IncRNA promotes neutrophil inflammatory activation in sepsis by supporting glycolytic metabolism with PFKFB3.

Cell Death and Disease (2021)12:1157; https://doi.org/10.1038/s41419-021-04428-7

\section{INTRODUCTION}

Sepsis is recognized as life-threatening organ dysfunction caused by the dysregulated host response to infection [1]. Despite the recent development of clinical therapeutic interventions, sepsis continues to be a major cause of death resulting from infection [1-3]. The pathogenesis of sepsis has not been well elucidated until now. Current efforts in understanding sepsis have indicated that metabolic reprogramming of innate immune cells plays a vital role in sepsis progression [4-6].

Neutrophils, the most abundant innate immune cells in human blood, reach the affected area of infection early to reduce the number of intruders and direct adaptive immune responses during sepsis [7]. However, excessive inflammatory neutrophil activation may lead to tissue damage and contribute to the development of organ dysfunction [8-10]. Recent studies have indicated that neutrophils undergo metabolic reprogramming to adapt to diverse disease conditions, such as sepsis, diabetes, and atherosclerosis [11-14]. Efforts to understand how neutrophils metabolic status is shifted may provide clues for developing better therapeutics for sepsis.
Several well-executed studies have suggested that glycolysis is the predominant metabolic pathway of neutrophil-mediated pathogen clearance [15-17]. Furthermore, glycolysis inhibition protected mice against enhanced neutrophilic responses, suggesting a direct connection of metabolic modulation during infection and inflammation [18]. 6-Phosphofructo-2-kinase/fructose-2,6biphosphatase 3 (PFKFB3) is a well-known regulator of glycolysis [19]. By mediating both the synthesis and degradation of fructose2,6-bisphosphate, PFKFB3 is required for cell proliferation and survival [20-22]. A recent study showed that blockade of the glycolytic activator PFKFB3 in tumor endothelial cells reduced cancer cell invasion, intravasation, and metastasis, and improved the effect of chemotherapy on primary and metastatic tumors [23]. However, little is known about the role of PFKFB3 in regulating neutrophil metabolism and function. Long noncoding RNAs (IncRNAs) are longer than 200 nucleotides and contribute to transcriptional control and posttranscriptional processing functions [24]. By interacting with mate RNA molecules, IncRNAs could potentially participate in modulation of mRNA stability control and translation activation [25-27]. Accumulating evidence has

\footnotetext{
${ }^{1}$ Department of Critical Care Medicine, Affiliated Hospital of Jiangsu University, Zhenjiang, China. ${ }^{2}$ Department of Critical Care Medicine, Jurong Hospital Affiliated to Jiangsu University, Zhenjiang, China. ${ }^{3}$ Department of Laboratory Medicine, Affiliated People's Hospital of Jiangsu University, Zhenjiang, China. ${ }^{4}$ Department of Electrocardiograph, The No. 4 Affiliated

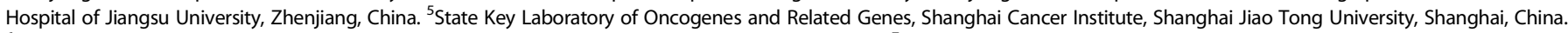

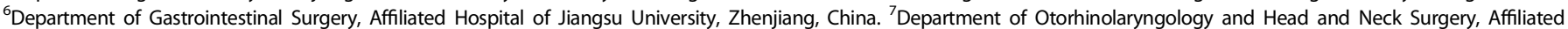

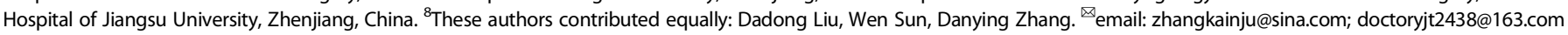
Edited by Dr Nirmal Robinson
}

Received: 22 May 2021 Revised: 3 November 2021 Accepted: 17 November 2021

Published online: 14 December 2021 
revealed the critical role that IncRNAs play to influence cellular metabolism in cell proliferation, activation, and apoptosis [28-30]. However, whether IncRNAs can manipulate neutrophil metabolic reprogramming in sepsis remains unclear.

In the present study, we found that the expression of G-quadruplex forming sequence-containing IncRNA (GSEC) in neutrophils was significantly increased in septic patients and positively correlated with PFKFB3 mRNA expression. In addition, in vitro studies confirmed that GSEC upregulated PFKFB3 transcription, and that glycolytic metabolism with PFKFB3 involvement further contributed to neutrophil inflammatory factor production. Our findings offer novel insight into the immune disorders in sepsis and may facilitate the exploration of potential therapeutic targets.

\section{RESULTS \\ Identification of differentially expressed IncRNAs and mRNAs in immunocytes of septic patients}

Aberrant transcriptional status is a hallmark of immune disorder in sepsis [31]. To identify the differentially expressed genes of immunocytes in septic patients, three sets of gene expression data (GSE64457, GSE28750, and GSE13904) were downloaded from the Gene Expression Omnibus (GEO) database for analysis in this study [32-34]. Total RNA of septic patients and healthy volunteers was independently extracted from blood neutrophils (GSE64457) and blood leukocytes (GSE28750 and GSE13904).

A hierarchical cluster analysis of each dataset was performed to obtain an overview of the expression profile of differentially expressed genes. The heat maps generated from the results showed a distinct regulatory direction and a clear separation between the differentially expressed IncRNAs in the sepsis samples and matched control samples (Fig. 1A and Supplementary Figs. 1A and 2A) and the differentially expressed mRNAs in the sepsis and control samples (Fig. 1B and Supplementary Figs. 1B and 2B). Next, the differentially expressed genes between sepsis samples and matched control samples were analyzed based on the following criteria: fold change $>1.2$ or $<-1.2$, a false discovery rate $($ FDR) $<0.05$, and a $P$-value $<$ 0.05 [35]. We found that 1733 IncRNAs (431 in GSE64457, 722 in GSE28750, and 580 in GSE13904) and 16,181 mRNAs (3498 in GSE64457, 6610 in GSE28750, and 6073 in GSE13904) were differentially expressed in septic patients.

Then, a Venn plot was generated to calculate the number and proportion of the differentially expressed genes in the three datasets. We obtained 33 overlapping IncRNAs (Fig. 1C and Supplementary Table 1), including 21 upregulated and 12 downregulated, and 878 overlapping mRNAs (Fig. 1D and Supplementary Table 2), including 464 upregulated and 414 downregulated. Interestingly, we noticed that, among the upregulated genes, TLR2, TLR5 and interleukin (IL) receptorassociated kinase 3 have been extensively studied in the regulation of inflammation and immune function [36-38]. In addition, metabolism-related genes, including PFKFB3, PRKAA1, $P Y G L$, and GYG1, were also upregulated, which indicated their potential roles in mediating immune responses in sepsis [39-41].

\section{LncRNA-mRNA co-expression analysis and functional annotation}

Increasing evidence confirms that IncRNAs play important roles in regulating the expression of protein-coding genes and, therefore, identifying co-expressed protein-coding genes may help in the assessment of IncRNA functions [42]. Therefore, IncRNA-mRNA coexpression analysis was performed to explore the relationship between IncRNAs and mRNAs in sepsis.

We calculated the Pearson's correlation for each pair of genes and defined the significantly correlated pair as the absolute value of any correlation coefficient greater than 0.75 . A total of 8834 differential IncRNA-mRNA pairs (1439 in GSE64457, 5620 in GSE28750, and
1778 in GSE13904) and 4150 differential mRNA-mRNA pairs (718 in GSE64457, 2483 in GSE28750, and 949 in GSE13904) were identified in the 3 datasets. Among these differentially expressed pairs, 372 differential overlapping pairs (including 166 IncRNA-mRNA pairs and 206 mRNA-mRNA pairs) were used to construct a co-expression network (Fig. 1E). From the co-expression network analysis, we obtained 11 IncRNAs (7 upregulated and 4 downregulated) (Supplementary Table 3) and 105 mRNAs (93 upregulated and 12 downregulated) (Supplementary Table 4).

Gene Ontology (GO) biological process analysis was performed based on the 105 co-expressed mRNAs [43]. With the criteria of an FDR $<0.05$ and a $P$-value $<0.01$, we obtained 54 biological processes, including 35 upregulated processes (Supplementary Table 5) and 19 downregulated processes (Supplementary Table 6). Among these processes, 22 upregulated biological processes (enriched with 39 mRNAs) were related to the immune response (Supplementary Fig. 3A and Supplementary Table 7) and metabolic processes (Supplementary Fig. 3B and Supplementary Table 8). Glucose is the basic nutrient in cells that is required to maintain normal survival and function. A growing body of evidence suggests that activated immunocytes generate energy in large part by upregulating aerobic glycolysis [44-46]. In this study, we found that the glucose metabolic process was upregulated, and that six co-expressed mRNAs (GYG1, MAPK14, PFKFB3, PGK1, PRKAA1, and PYGL) were enriched in this process (Supplementary Fig. 3B and Supplementary Table 8). In addition, other significantly enriched biological processes were involved in cell differentiation and gene transcription (Supplementary Fig. 3C and Supplementary Tables 5 and 6).

A Kyoto Encyclopedia of Genes and Genomes (KEGG) pathway analysis was performed to determine the significantly changed pathways in which the co-expressed mRNAs are involved [47]. Based on the criteria of an FDR $<0.05$ and a $P$-value $<0.01$, we identified 15 upregulated pathways and 1 downregulated pathway in immunocyte dysfunction in septic patients (Supplementary Table 9). Consistent with our findings in the GO biological process analysis, among the upregulated pathways, 11 pathways (enriched with 21 mRNAs) were found to be related to the immune response (Supplementary Fig. 4A and Supplementary Table 10) and metabolic processes (Supplementary Fig. 4B and Supplementary Table 11). The adenosine monophosphate-activated protein kinase pathway, which is extensively involved in cellular energy homeostasis, was also upregulated and associated with three coexpressed mRNAs (CAB39, PFKFB3, and PRKAA1) in sepsis [48]. These results suggested that immunocytes of septic patients undergo metabolic reprogramming.

\section{LncRNA-mRNA pathway network analysis}

To explore the specific IncRNA-mRNA interaction in sepsis-related metabolism reprogramming, IncRNA-mRNA pathway networks were constructed. The results showed that 6 upregulated IncRNAs and 27 associated upregulated mRNAs were involved in 15 upregulated pathways (FDR $<0.05$ and $P<0.01$ ) (Fig. 2). Among these IncRNAs, the NONHSAT160878.1 IncRNA was associated with the most mRNAs (degree $=20$ ). It was involved in glucose metabolism by associating with four glucose metabolism-related mRNAs (GYG1, MAPK14, PFKFB3, and PYGL).

The IncRNA NONHSAT160878.1 is located on chromosome 11 (126340958-126355587) and was identified as a GSEC. A previous study showed that GSEC can modulate colon cancer progression by promoting cell migration [49]. However, the biological role of GSEC in sepsis remains unclear. PFKFB3 is a well-known regulator of glycolytic metabolism and plays an important role in cell survival and activation [20-22]. Based on our analysis, GSEC and PFKFB3 were co-expressed in leukocytes of septic patients and associated with neutrophil glycolysis. However, whether a causal relationship exists between GSEC and the PFKFB3 genes remains to be determined. 


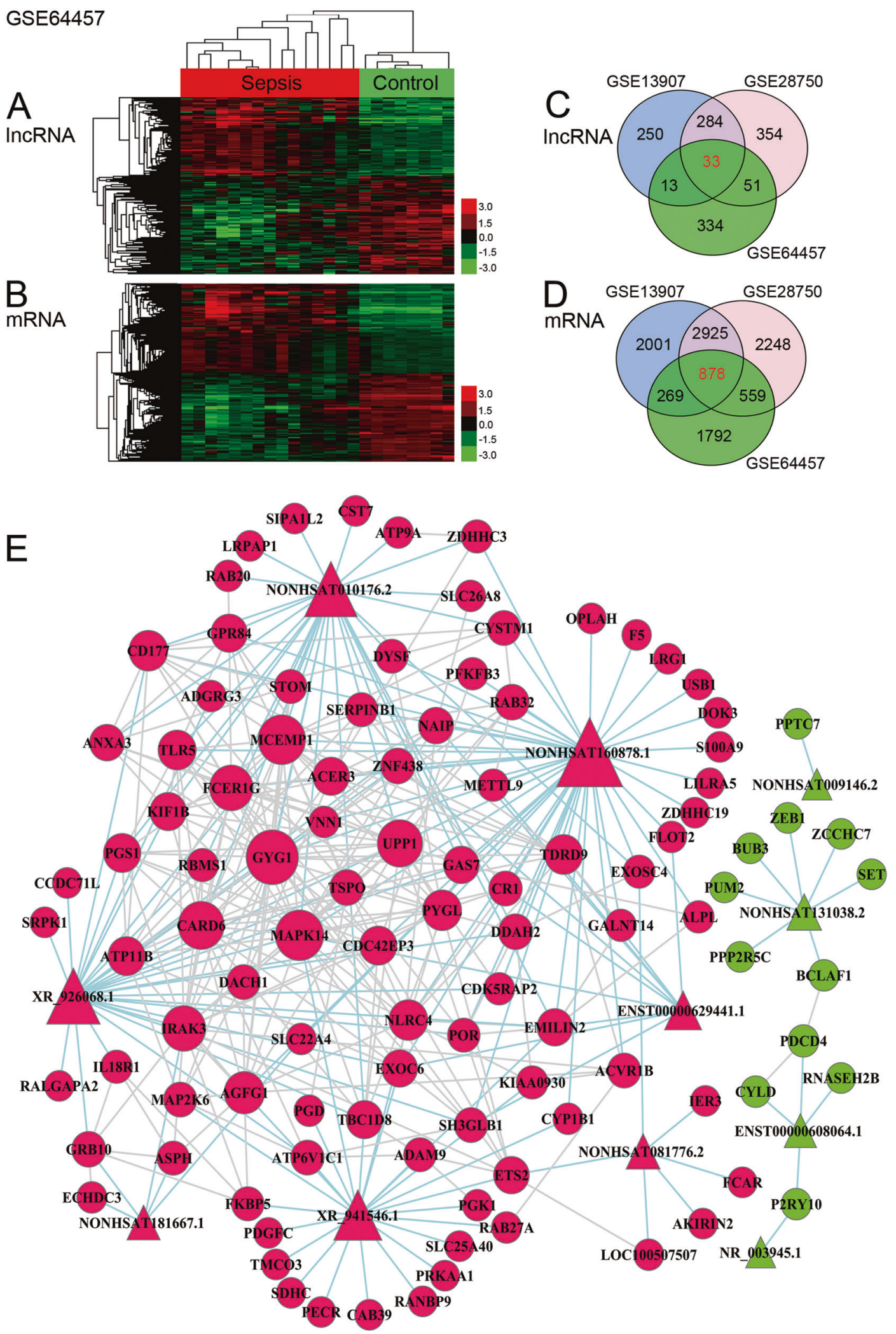

Fig. 1 Identification of differentially expressed IncRNAs and mRNAs in the immunocytes of septic patients. A Heat map of 431 differentially expressed IncRNAs (including 192 upregulated and 239 downregulated IncRNAs) identified in the microarrays in the GSE64457 dataset. B Heat map of 3498 differentially expressed mRNAs (including 1765 upregulated and 1733 downregulated mRNAs) identified in the microarrays in the GSE64457 dataset. C Venn plot of the 1319 differentially expressed IncRNAs in the 3 datasets. D Venn plot of the 10,672 differentially expressed mRNAs in the 3 datasets. E Co-expression network of the significantly differentially expressed IncRNAs and mRNAs. Circles indicate mRNAs and triangles indicate IncRNAs. Red represents upregulated expression and green represents downregulated expression. Baby blue lines indicate the interaction between IncRNAs and mRNAs, and gray lines indicate mRNAs interacting with other mRNAs. 


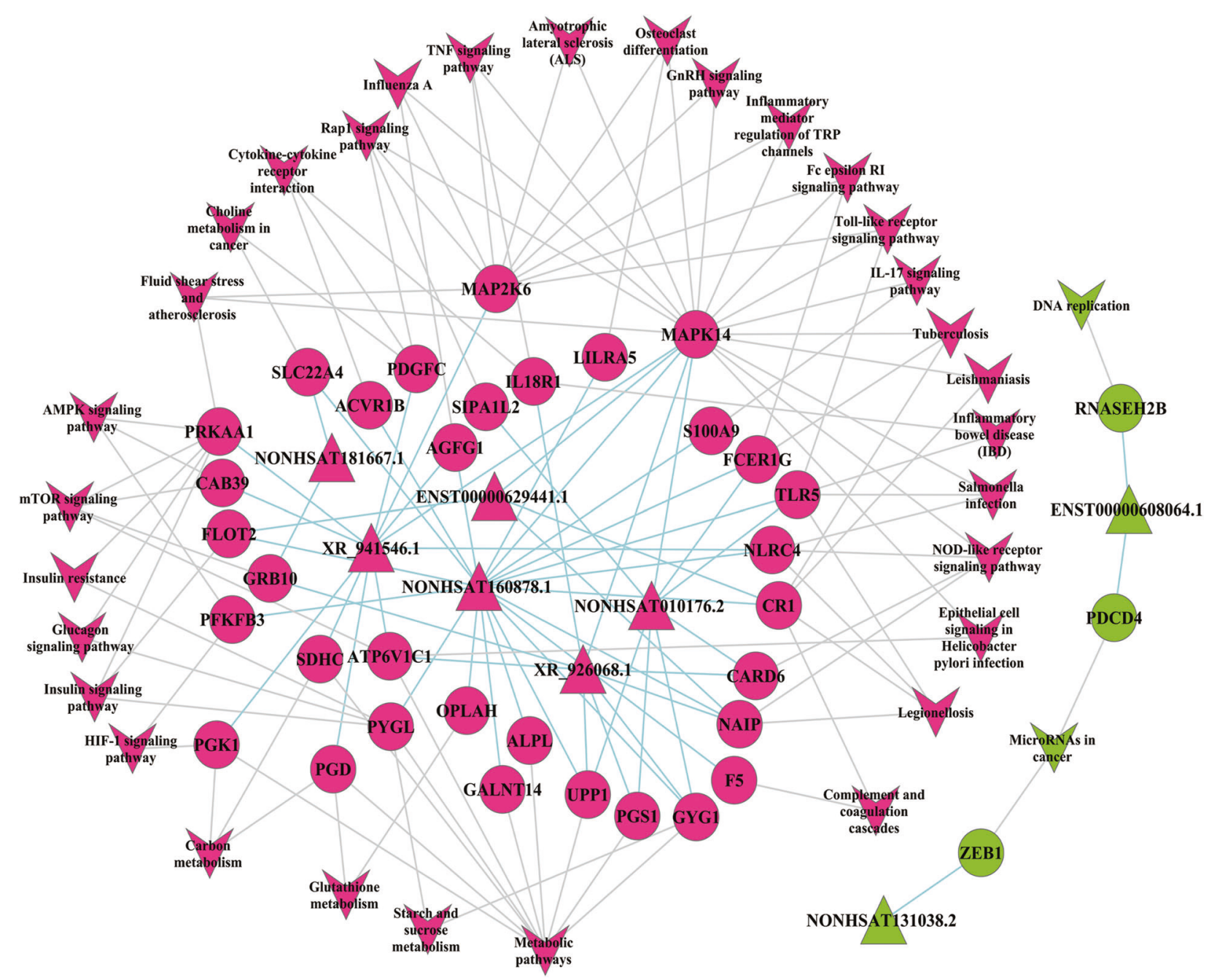

Fig. 2 Pathway network analysis of the significantly differentially expressed IncRNAs and mRNAs. The role of each pathway in the network was measured by counting its connections to upstream and downstream pathways. A pathway with a high degree value likely plays an important role in the signaling network. Circles indicate mRNAs and triangles indicate IncRNAs. Red represents upregulated pathways and green represents downregulated pathways. Baby blue lines indicate the interaction between IncRNAs and mRNAs, and gray lines indicate interactions between pathways.

\section{Expression and correlation of GSEC and PFKFB3 genes in septic neutrophils}

Neutrophils are the most abundant innate immunocytes and the first mediator to enter the affected area during infection [7]. However, in sepsis, overactivation of neutrophils contributes to systemic inflammation and is the main cause of organ dysfunction. To investigate whether neutrophil inflammatory activation is related to GSEC and/or PFKFB3 mRNA, primary neutrophils were obtained from the blood of septic patients and healthy volunteers, and the expression of GSEC and PFKFB3 was determined. The results showed that neutrophils in septic patients had significantly higher expression of GSEC and PFKFB3 than those in healthy volunteers (Fig. 3A, B). Pearson's correlation analysis showed that GSEC was positively correlated with PFKFB3 mRNA $(r=0.74, P<$ 0.01) (Fig. $3 C$ ), which was consistent with the results obtained from the analysis of the three GEO datasets (Supplementary Fig. 5). In addition, we found that the mRNA expression of inflammatory cytokine tumor necrosis factor (TNF)-a was upregulated in septic neutrophils (Fig. 3D). Another correlation analysis indicated that TNF-a mRNA expression was positively correlated with GSEC (Fig. 3E) and PFKFB3 (Fig. 3F). Similar results were found for IL-1 $\beta$ (Supplementary Fig. 6) and IL-6 (Supplementary Fig. 7) expression in septic neutrophils. These results suggest the potential interaction between GSEC and PFKFB3 in neutrophil inflammation activation during sepsis.

\section{GSEC is essential for PFKFB3 expression in inflammatory neutrophils}

We examined whether the sepsis-associated inflammatory stimuli lipopolysaccharide (LPS) and TNF-a regulate the expression of GSEC and PFKFB3 mRNA in neutrophils in vitro. We found that LPS and TNF-a stimulation markedly increased the expression levels of the GSEC and PFKFB3 in primary human neutrophils compared with that in the control group (Fig. 4A-C and Supplementary Figs. 8A and 9A, B). Similar results were found in neutrophil-like differentiated $\mathrm{HL}-60$ (dHL-60) cells (Fig. 4D-F and Supplementary Figs. 8B and 9C, D), in vitro neutrophil models extensively used by our group [50] and other researchers [51].

Recent studies have indicated the essential role of IncRNAs in regulating mRNA expression [52]. As primary neutrophils cannot be genetically manipulated, $\mathrm{dHL}-60$ cells were used to determine whether GSEC can influence the expression of PFKFB3. We used lentiviral vectors to generate $\mathrm{dHL}-60$ cells with stable GSEC knockdown or overexpression (OE), and an empty vector was used as the control. We found that LPS stimulation significantly increased the expression of PFKFB3 in the empty vector-transfected dHL-60 

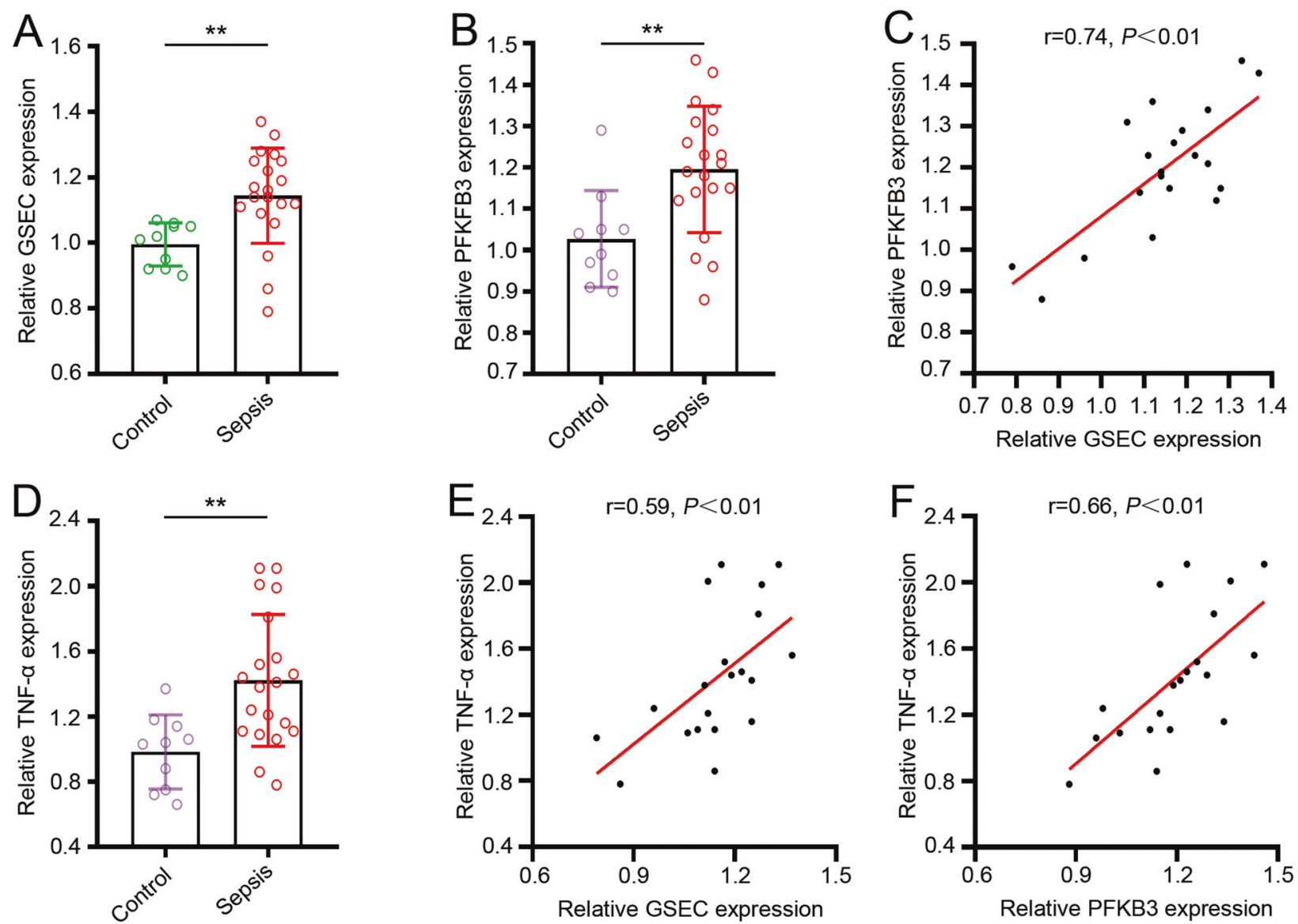

Fig. 3 Expression and correlation of GSEC, PFKFB3, and inflammatory cytokine genes in septic neutrophils. A Expression of GSEC in septic neutrophils. B Expression of PFKFB3 in septic neutrophils. C Correlation of GSEC and PFKFB3 genes in septic neutrophils. D Expression of TNF$\alpha$ in septic neutrophils. E Correlation of GSEC and TNF- $\alpha$ in septic neutrophils. F Correlation of PFKFB3 and TNF- $\alpha$ in septic neutrophils. For each group: septic patients, $n=20$; healthy volunteers, $n=10$, three independent experiments. Statistics were calculated using Student's $t$-test $(\mathbf{A}, \mathbf{B}, \mathbf{D})$ or Pearson's analysis $(\mathbf{C}, \mathbf{E}, \mathbf{F})$.

cells. However, in the GSEC-knockdown cells, the expression of PFKFB3 was significantly reduced (Fig. 4G, $\mathrm{H}$ and Supplementary Figs. $8 \mathrm{C}$ and $9 \mathrm{E}, \mathrm{F})$. In contrast, LPS-enhanced PFKFB3 expression was further increased in the GSEC-overexpressing dHL-60 cells (Fig. 4l, J and Supplementary Figs. 8D and 9G, H). However, these reductions were not observed in GSEC-knockdown dHL-60 cells without LPS stimulation (Supplementary Fig. 10).

\section{GSEC was essential for enhancing PFKFB3 mRNA stability}

Dual-luciferase reporter assay was used to investigate whether GSEC IncRNA could regulate PFKFB3 mRNA transcription. Results indicated that GSEC IncRNA can positively regulate PFKFB3 transcription through two different regions of PFKFB3 (Fig. 5A, B). A previous study revealed that IncRNAs can regulate mRNA expression by influencing mRNA stability [53]. Thus, we next sought to determine whether the GSEC can influence the stability of PFKFB3 mRNA. Using actinomycin $D$ to block transcription, we observed that PFKFB3 mRNA expression was gradually reduced in LPS-stimulated dHL-60 cells (Fig. 5C, D). The GSEC-knockdown cells showed a faster rate of PFKFB3 mRNA decay than the control cells (Fig. 5C), whereas the GSECoverexpressing cells showed an attenuated rate of PFKFB3 mRNA decay (Fig. 5D). In addition, we found that lentiviral vector stimulation did not influence the stability of PFKFB3 $m R N A$, and that GSEC OE did not reduce the rate of GSEC IncRNA decay in dHL-60 cells (Supplementary Fig. 11). These data indicated that GSEC was essential for supporting PFKFB3 expression in inflammatory dHL-60 cells by enhancing PFKFB3 mRNA stability.

\section{GSEC/PFKFB3-supported glycolytic metabolism enhances} dHL-60 cell inflammatory factor expression

To investigate whether glycolytic metabolism was regulated by the effects of GSEC on PFKFB3 expression, the extracellular acid ratio (ECAR) was measured in $\mathrm{dHL}-60$ cells. The lentiviral vectors did not affect the ECAR in LPS-stimulated dHL-60 cells (Supplementary Fig. 12). After LPS stimulation, the GSEC-knockdown dHL60 cells showed a lower ECAR than the control dHL-60 cells, whereas a higher ECAR was observed in the GSEC-overexpressing dHL-60 cells (Fig. 6A, B). This result indicated that GSEC was essential for supporting neutrophil glycolysis. To further investigate whether GSEC reprograms the glycolytic metabolism in neutrophils through PFKFB3 mRNA. PFK15, a small molecule inhibitor of PFKFB3, was used to inhibit PFKFB3 enzyme activity in $\mathrm{dHL}-60$ cells. The results showed that PFK15 markedly reduced the ECAR in the dHL-60 cells. Similar ECARs were detected in the PFK15-treated GSEC-knockdown and GSEC-overexpressing dHL-60 cells. These results indicated that PFKFB3 expression regulated by GSEC was involved in glycolytic reprogramming in dHL-60 cells.

Next, we explored whether inflammatory cytokine expression is regulated by GSEC through glycolysis involving PFKFB3 expression. First, we found that lentiviral vectors did not affect TNF-a expression in $\mathrm{dHL}-60$ cells (Supplementary Fig. 13A). After LPS stimulation, we found that TNF-a expression was markedly upregulated compared with that in unstimulated cells. Then, TNF-a expression was measured in GSEC-knockdown and GSEC-overexpressing dHL-60 cells. We observed that GSEC-knockdown dHL-60 cells expressed a lower level of TNF-a mRNA than LPS-stimulated vehicle cells and TNF-a mRNA 

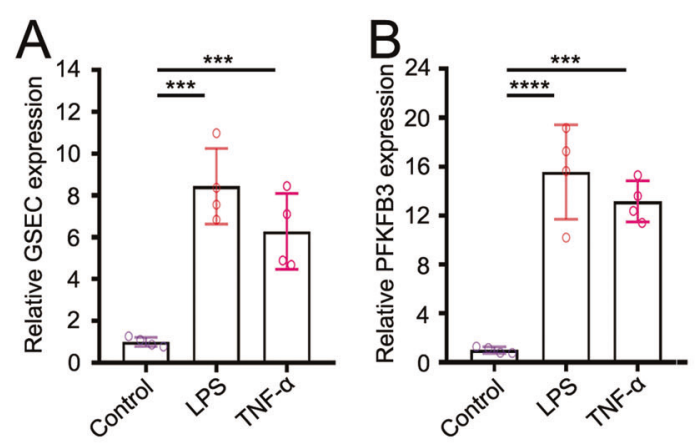

C

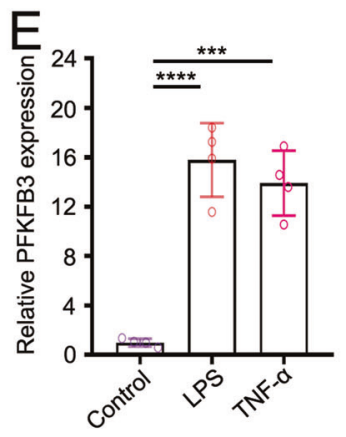

$\mathrm{F}$

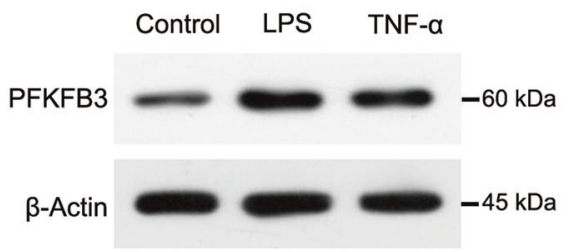

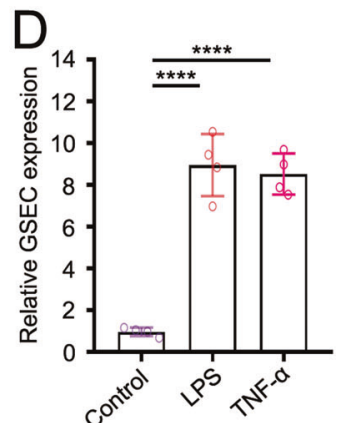

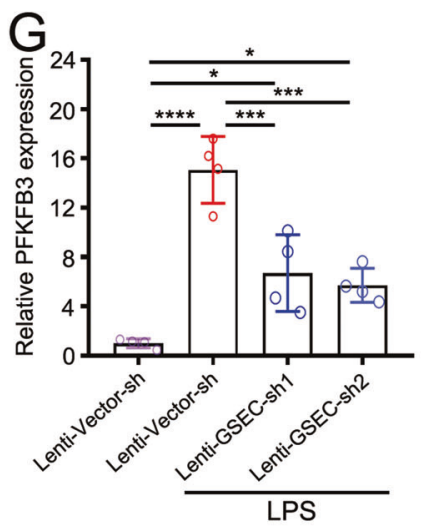

$J$

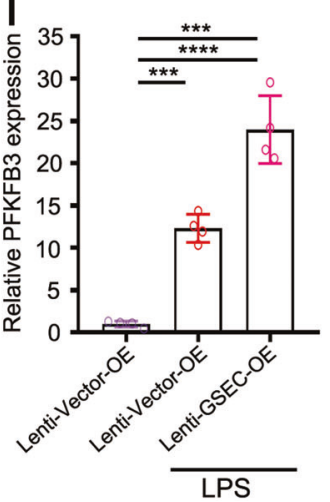

J

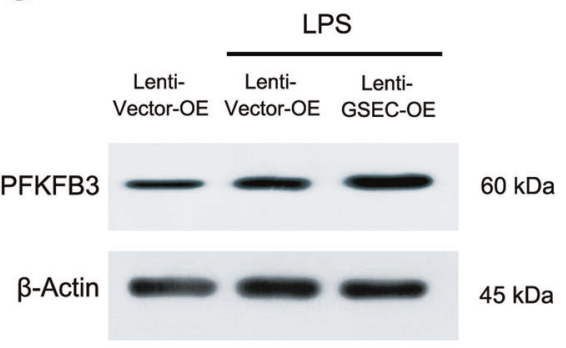

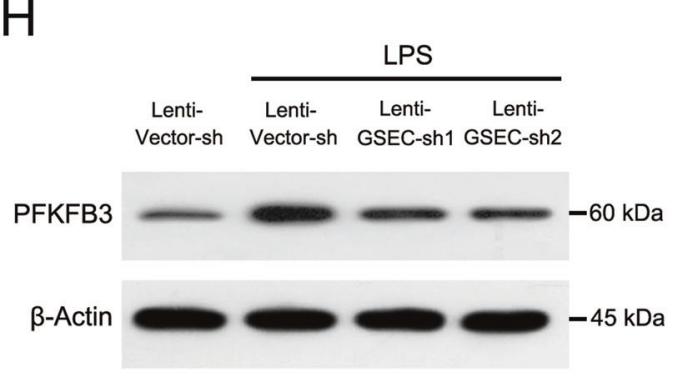

Fig. 4 GSEC is essential for PFKFB3 expression in inflammatory neutrophils. A The expression level of GSEC in primary human neutrophils. B The expression level of PFKFB3 in primary human neutrophils. C Western blot analysis of PFKFB3 in primary human neutrophils. D The expression level of the GSEC in dHL-60 cells. E The expression level of PFKFB3 in dHL-60 cells. F Western blot analysis of PFKFB3 in dHL-60 cells. G The level of PFKFB3 expression in GSEC-knockdown dHL-60 cells. H Western blot analysis for PFKFB3 in GSEC-knockdown dHL-60 cells. I The level of PFKFB3 expression in GSEC-overexpressing dHL-60 cells. J Western blot analysis of PFKFB3 in GSEC-overexpressing dHL-60 cells. Neutrophils and dHL-60 cells were treated LPS or TNF- $\alpha$ for $12 \mathrm{~h}$. Statistics were calculated using one-way ANOVA with Tukey's post hoc tests. ${ }^{*} P<0.05,{ }^{* *} P<0.01,{ }^{* * *} P<0.001,{ }^{* * * *} P<0.0001$.

expression level in PFK15-inhibited cells was reduced to a level similar to that of the GSEC-knockdown dHL-60 cells (Fig. 6C). PFK15 failed to further reduce the expression of TNF-a mRNA in the GSECknockdown cells. In contrast to our findings with GSEC-knockdown cells, we observed that TNF-a mRNA expression was higher in GSECoverexpressing cells (Fig. 6D). PFK15 inhibited the expression of TNF-a mRNA in vector cells and GSEC-overexpressing cells. 2-Deoxy-Dglucose (2-DG), an inhibitor of hexokinase II, was used to block glycolysis in $\mathrm{dHL}-60$ cells. The results showed that 2-DG significantly inhibited TNF-a mRNA expression (Fig. 6C, D), which indicated that glycolysis supported inflammatory cytokine mRNA expression in activated $\mathrm{dHL}-60$ cells. Furthermore, we found that 2-DG further reduced the mRNA expression of TNF-a in the GSEC-knockdown and GSEC-overexpressing cells stimulated by LPS (Fig. 6C, D and Supplementary Fig. 14A, B). The level of TNF-a protein was similar, as determined by enzyme-linked immunosorbent assay (ELISA)
(Fig. 6E, F and Supplementary Figs. 13B and 14C, D). Furthermore, we wondered whether inflammatory $\mathrm{IL}-1 \beta$ and $\mathrm{IL}-6$ expression is regulated by GSEC through glycolytic metabolism involving PFKFB3. The expression of IL-1 $\beta$ (Supplementary Figs. 15 and 16) and IL- 6 (Supplementary Figs. 17 and 18) was similar to that of TNF-a. These results suggested that GSEC-elevated inflammatory cytokine expression is PFKFB3 dependent. Together, our findings indicated that GSEC/PFKFB3-supported glycolytic metabolism promoted inflammatory cytokine expression in $\mathrm{dHL}-60$ cells.

\section{DISCUSSION}

The proinflammatory response in the acute phase of sepsis eliminates invading pathogens and involves immunocyte activation and subsequent production of cytokines such as TNF- $a, \mathrm{IL}-1 \beta$, and IL-6 [54]. However, a hyperactive proinflammatory response 
A

PFKFB3-wt1: 3' - ttctgg......acaaagaa......aattacact- 5' \|\|\|\|

PFKFB3-mut1: 3' - ttctgg......gagggtcc.......aattacact- 5'

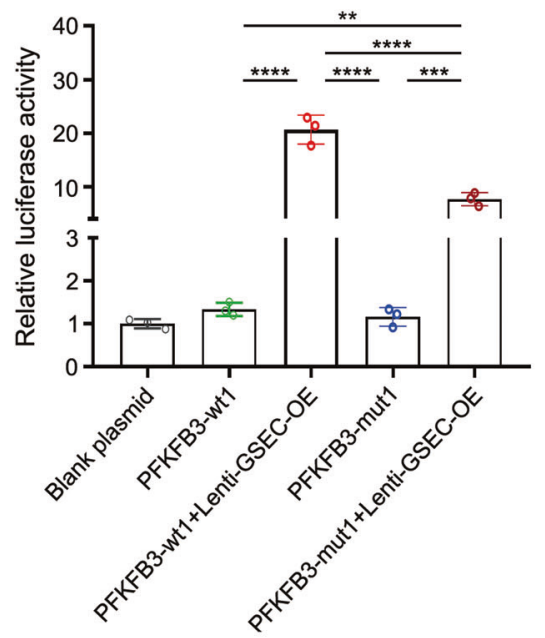

C

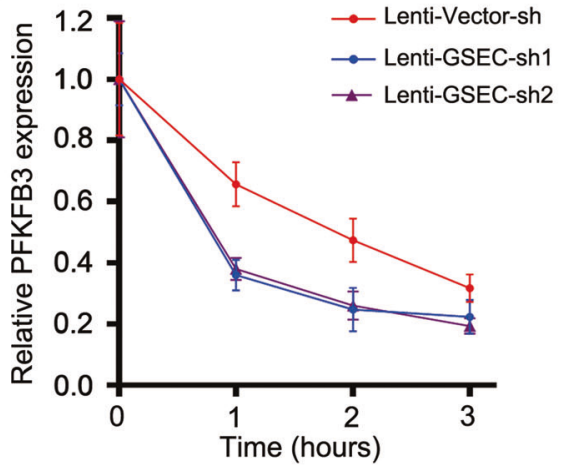

B
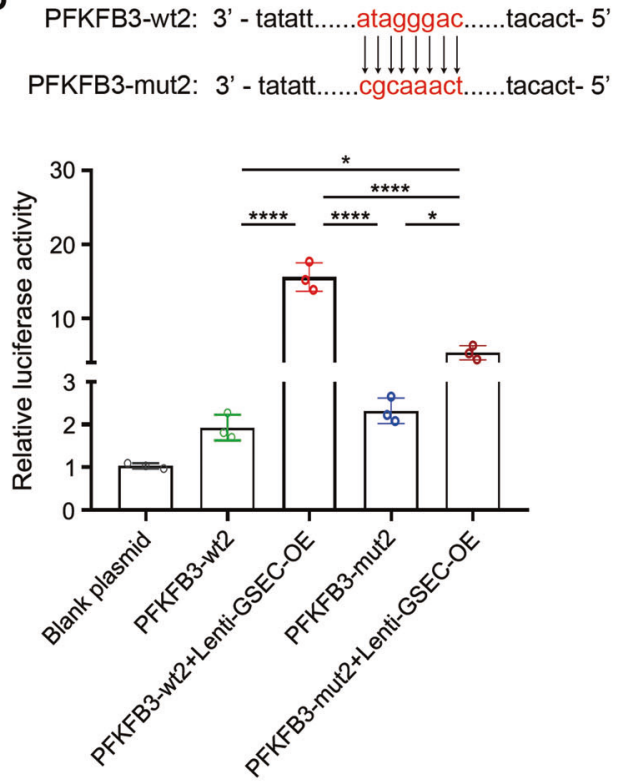

D

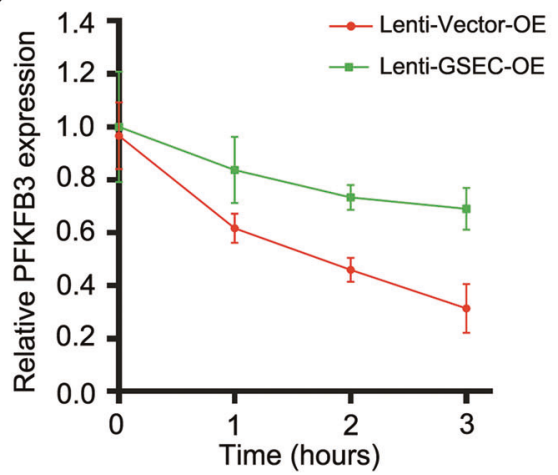

Fig. 5 GSEC was essential for enhancing PFKFB3 mRNA stability. A GSEC positively regulates PFKFB3 transcription through the active site (acaaagaa) of PFKFB3. B GSEC positively regulates PFKFB3 transcription through the active site (ataggac) of PFKFB3. C The stability of PFKFB3 expression in GSEC-knockdown dHL-60 cells. D The stability of PFKFB3 expression in GSEC-overexpressing dHL-60 cells. The dHL-60 cells were treated LPS for $12 \mathrm{~h}$. Statistics were calculated using one-way ANOVA with Tukey's post tests. ${ }^{*} P<0.05,{ }^{*} P<0.01,{ }^{* * *} P<0.001,{ }^{* * * *} P<0.0001$.

may exert detrimental effects on the host by eliciting high fevers, hypotension, and organ failure [55]. Therefore, to develop effective therapeutic strategies for improving patient prognosis after sepsis, it is crucial to understand the precise molecular mechanisms of sepsis-induced immunocyte activation. Recent studies have shown that numerous metabolic changes in immunocytes appear to be vital for immune system dysfunction and sepsis progression [5,56-58]. A typical metabolic change is observed in the hyperinflammatory phase of sepsis and is accompanied by a metabolic switch from oxidative phosphorylation to glycolytic metabolism in many immunocytes [58-61]. Our bioinformatics analyses of three independent GEO datasets confirmed that glycolytic metabolism and associated pathways were activated in blood immunocytes. Because of the crucial role of glycolysis in immunocyte proinflammatory activation [62], we speculate that further investigation into the metabolism reprogramming mechanism in immunocyte during sepsis may facilitate the identification of potential therapeutic targets.

Glycolytic metabolism (also termed glycolysis) begins with the uptake of extracellular glucose and subsequent processing of glucose in the cytosol to eventually yield adenosine triphosphate (ATP) and numerous other products [16]. Previous studies have shown that enhanced glycolysis enables immunocytes to generate sufficient ATP and biosynthetic intermediates to carry out its particular effector functions, including antigen presentation and inflammatory cytokine production [58, 63-65]. In addition, recent advances experiments based on animal models of sepsis have revealed that decreases in glycolysis can inhibit the release of proinflammatory cytokines and thus improve the survival outcomes of the animal models [66]. Consistent with these previous studies, our results confirmed that LPS stimulation increased the level of ECAR, a key indicator of glycolysis, in dHL-60 cells. Therefore, investigating the mechanism of sepsis-induced glycolysis may provide a scientific basis for sepsis treatment.

Other studies into the mechanism have revealed that genes encoding key enzymes associated with glycolysis are upregulated in human leukocytes during the initial hyperinflammation induced by endotoxin [67]. In agreement with this study on leukocytes, our results showed that during the acute phase of sepsis, the expression level of PFKFB3 mRNA was upregulated in primary human neutrophils. PFKFB3 is a key regulatory glycolytic enzyme involved in glucose decomposition into ATP and has dual kinase and phosphatase activities [19]. PFKFB3 kinase activity is known to increase the rate of glycolysis and promote the proliferation, migration, invasion, and growth of tumor cells [68-70]. However, recent studies have revealed another role for PFKFB3: PFKFB3- 

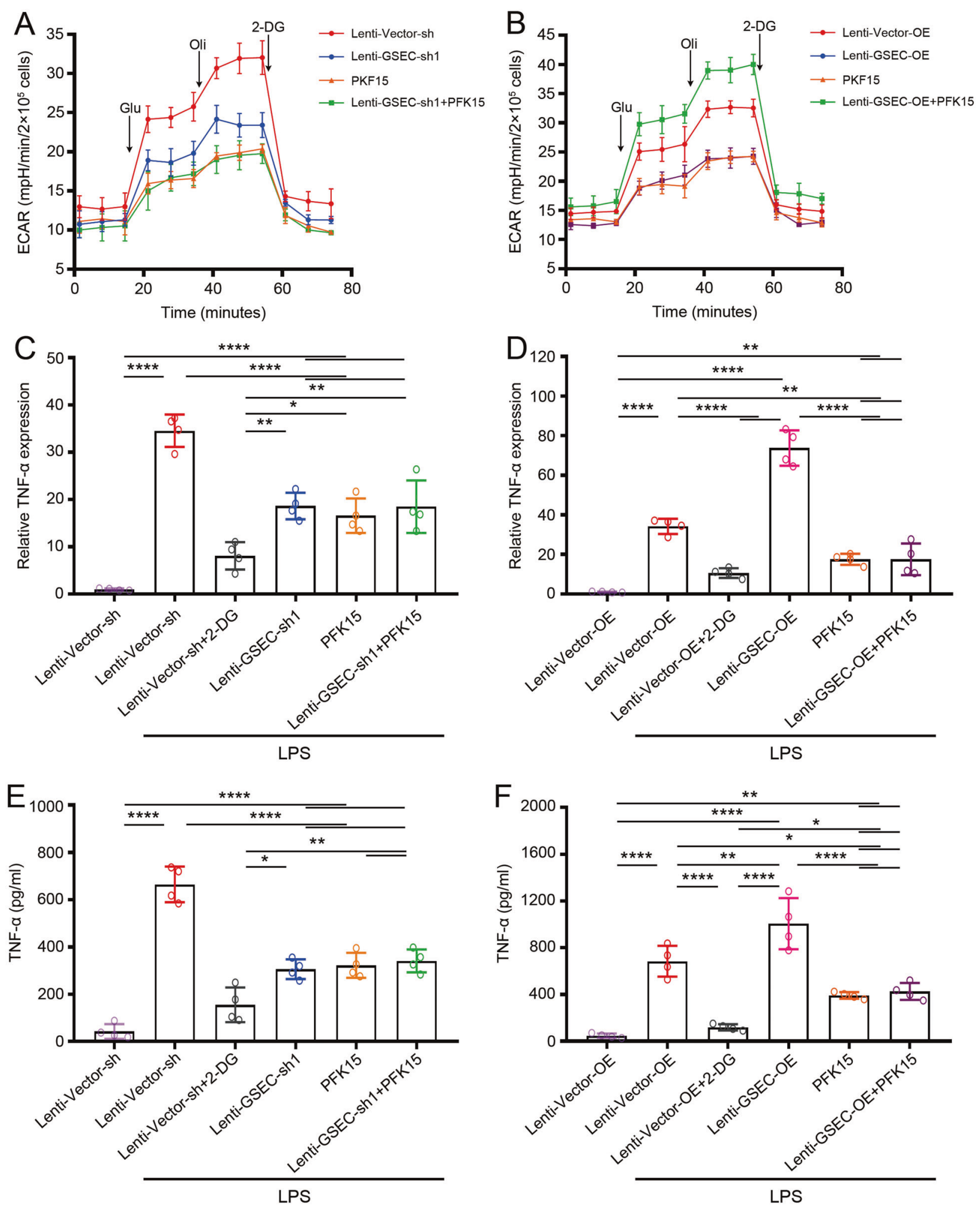

Fig. 6 GSEC/PFKFB3-supported glycolytic metabolism enhances dHL-60 cell inflammatory factor expression. A The ECAR was detected in GSEC-knockdown dHL-60 cells. B The ECAR was detected in GSEC-overexpressing dHL-60 cells. C The level of TNF- $\alpha$ mRNA expression was detected in GSEC-knockdown dHL-60 cells. D The level of TNF- $\alpha$ mRNA expression was detected in GSEC-overexpressing dHL-60 cells. E The level of TNF- $\alpha$ protein was detected in GSEC-knockdown dHL-60 cells. F The level of TNF- $\alpha$ protein was detected in GSEC-overexpressing dHL60 cells. For all experiments: $n=4$ per group, three independent experiments. Neutrophils and dHL-60 cells were treated LPS for $12 \mathrm{~h}$. ${ }^{*} P<0.05,{ }^{* *} P<0.01,{ }^{* * *} P<0.001,{ }^{* * *} P<0.0001,{ }^{n s} P$ indicates no statistical significance. Glu, glucose; Oli, oligomycin; 2-DG, 2-deoxyglucose. 
mediated glycolysis is involved in the regulation of the inflammatory response. Jiang et al. [71] found that PFKFB3 promotes the antiviral capacity of macrophages by metabolically supporting the uptake and elimination of virus-infected cells. Zhang et al. [72] demonstrated that TNF- $a$ stimulation increased endothelial PFKFB3 expression and PFKFB3 knockdown almost completely blocked the TNF-a-induced release of proinflammatory cytokines. Similar to these studies, our study showed that both TNF-a and LPS stimulation can significantly increase the expression of PFKFB3 in primary human neutrophils. In addition, we found that the PFKFB3 expression is increased in the neutrophils of septic patients. Some recently published studies have shown that blocking PFKFB3 protects mice from acute lung injury by suppressing hyperinflammation [73-75]. Consistent with these studies, our study also revealed that inhibiting PFKFB3 enzyme activity with PFK15 markedly reduced the rate of glycolysis and the expression of TNF- $\alpha, I L-1 \beta$, and IL- 6 mRNAs and protein in dHL- 60 cells. All of these results demonstrate that the glycolytic enzyme PFKFB3 plays a critical role in sepsis-induced neutrophil activation.

In addition to known mRNAs, IncRNAs are thought to be involved in glycolytic reprogramming [29, 76-78]. LncRNAs are widely expressed during immunocyte generation, differentiation, and activation, and they can control important aspects of immunity [79]. A few IncRNAs have been implicated in immunocyte glycolysis regulation, but the underlying mechanisms remain poorly understood. Liu et al. [80] found that the IncRNA AGPG plays a pivotal role in glycolysis in esophageal squamous cell carcinoma by directly binding and regulating PFKFB3. In agreement with this study, our results showed that the expression level of GSEC was significantly increased in sepsis-induced neutrophils and it influenced glycolysis in $\mathrm{dHL}-60$ cells by directly enhancing PFKFB3 mRNA transcription and translation. We also verified that this GSEC regulates the transcription of PFKFB3 through different regions of PFKFB3. Considering the proinflammatory role of IncRNAs, we investigated the expression of inflammatory factors in dHL-60 cells exposed to LPS. We found that GSEC/PFKFB3supported glycolytic metabolism enhances dHL-60 cell inflammatory factor expression.

In conclusion, our study showed that GSEC plays a pivotal role in promoting glycolysis in neutrophils by enhancing PFKFB3 transcription and translation, facilitating neutrophil inflammatory factors production during the acute phase of sepsis (Supplementary Fig. 19). Therefore, our study suggested that GSEC-regulated PFKFB3-mediated glycolytic reprogramming is a potential sepsis therapeutic target. Notably, the method we used to isolate human neutrophils did not eliminate eosinophils. However, as the eosinophil proportion was found to be $<1 \%$ of the cell population analyzed, we suggested that this proportion might have no significant influence on our main findings.

\section{MATERIALS AND METHODS Materials}

LPS (Escherichia coli O55:B5), dimethylsulfoxide (DMSO), fetal bovine serum (FBS), and TNF-a were obtained from Sigma-Aldrich (St. Louis, MO, USA). Hanks' balanced salt solution (HBSS) and RPMI-1640 medium were obtained from Thermo Scientific (Waltham, MA, USA). TRIzol reagent and a RNeasy kit were obtained from Qiagen (Dusseldorf, NRW, Germany). ELISA kits for IL-1 $\beta$, TNF- $\alpha$, and IL- 6 detection were obtained from Qiaoy (Shanghai, China). Rabbit anti-PFKFB3 monoclonal antibody and rabbit anti- $\beta$-actin monoclonal antibody were obtained from CST (Boston, MA, USA). GSEC-knockdown and OE lentivirus vectors were constructed by GeneChem (Shanghai, China). A NanoDrop ND-1000 spectrophotometer was obtained from Thermo Fisher Scientific (Waltham, MA, USA). A Seahorse XF96 Flux Analyzer was obtained from Agilent Technologies (Palo Alto, CA, USA). SYBR Premix Ex Taq was obtained from Roche Life Science (Basel, KB, Switzerland).

\section{GEO database retrieval}

We systematically retrieved public microarray datasets by using the key word "sepsis" in the GEO database (https://www.ncbi.nlm.nih.gov/geo/). Datasets were selected according to the following criteria: the data referred to (1) human genes; (2) studies with case control(s); (3) neutrophils (at least those in whole blood) obtained from participants and used for gene expression analysis; and (4) details of gene expression.

After careful filtering, three sets of gene expression data (GSE13904, GSE28750, and GSE64457) were included in this study [32-34]. We included data of 50 leukocyte samples (including 18 samples from control subjects and 32 samples from septic patients (day 1) from the GSE13904 dataset, 30 leukocyte samples (including 20 samples from control subjects and 10 samples from septic patients) from the GSE28750 dataset, and 23 neutrophil samples (including 8 samples from control subjects and 15 samples from septic patients) from the GSE64457 dataset.

Identification of annotated and differentially expressed genes Raw CEL files of the three datasets were downloaded from the GEO database and then preprocessed (background correction, quantile normalization, and log2 transformation) using the robust multichip average method with the R package "affy" [81]. Next, hybridization probes were mapped to genes (Entrez Gene IDs) according to the platform table (GPL570). In addition, to profile the gene expression in the data, the expression levels of the genes in the downloaded datasets were compared with those in the NCBI RefSeq transcript (mRNA and IncRNA), Ensembl (InCRNA), and NONCODE 2016 (IncRNA) databases using the BLAST program. The workflow of probe set annotation is summarized in Supplementary Fig. 20. The differentially expressed genes in the septic patients were identified by hierarchical cluster analyses and Venn plots.

Co-expression network construction and enrichment analyses A gene co-expression network analysis was performed to elucidate the relationship between IncRNAs and mRNAs, and identify the key genes in septic patients by using the R package "WGCNA" [82]. A co-expression network was constructed by using Cytoscape 3.4.0 software [83]. To investigate the biological function and signaling pathways of mRNAs, DAVID (http://david.ncifcrf.gov) was used for GO annotation and KEGG pathway enrichment of co-expressed mRNAs [43, 47, 84]. A IncRNA-mRNA pathway network was created based on the results of the IncRNA-mRNA co-expression network and pathway analyses by using Cytoscape 3.4.0 software [83]

\section{Clinical settings}

A prospective case-control study was performed to evaluate the expression of genes in neutrophils in septic patients. Septic patients who were admitted to the intensive care unit (ICU) of the Affiliated Hospital of Jiangsu University between October 2018 and December 2019 were recruited. The inclusion criteria for septic patient inclusion were as follows: (1) age $\geq 18$ years; (2) clinical evidence of infection with sequential organ failure assessment (SOFA) score $\geq 2$. The exclusion criteria were as follows: (1) age $>80$ years; (2) patients whose immune system was suppressed (such as individuals with HIV, autoimmune disease, and/or cancer); (3) patients with hematopoietic disease; and (4) patients who received drugs within the last 2 weeks that might affect neutrophil function. A total of 20 septic patients were included in the present study.

Data including age, sex, SOFA score, Acute Physiology and Chronic Health Evaluation II score, absolute lymphocyte count, absolute neutrophil count, primary site of infection, pathogenic bacteria causing infection, and 28-day mortality were collected. Blood from septic patients was collected at admission to the ICU and before treatment of the infection. Ten healthy volunteers who underwent routine physical examinations in the hospital were recruited via clinical history and laboratory studies to serve as controls. There was no significant difference in the basic data (except absolute neutrophil count) between the two groups (Supplementary Table 12).

After written informed consent was obtained, blood specimens were extracted from the cubital veins of septic patients and healthy drug-free donors. This study was approved by the Medical Ethical Committee of Jiangsu University.

\section{Human neutrophil isolation and stimulation}

Human neutrophils were isolated from human peripheral blood byFicoll/ Hypaque centrifugation as previously described [50]. Briefly, human blood 
was collected and mixed with an equal volume of $3 \%$ dextran. The mixed blood was incubated for $30 \mathrm{~min}$ at room temperature and the supernatant was centrifuged with Ficoll/Hypaque for $30 \mathrm{~min}$. Then, the neutrophilcontaining pellet was resuspended in sterile $\mathrm{dd}_{2} \mathrm{O}$ water to facilitate erythrocyte lysis. Finally, the neutrophils were washed with HBSS and resuspended in RPMI-1640 with $1 \%$ heat-inactivated FBS. The purity (>97\%) of the neutrophils was measured by flow cytometry and adjusted on the basis of CD66b and Siglec-8 co-staining (Supplementary Fig. 21) [85-87]. The isolated neutrophils in RMPI-1640 culture medium were stimulated with LPS $(100 \mathrm{ng} / \mathrm{mL}$, Sigma-Aldrich) and TNF-a $(10 \mathrm{ng} / \mathrm{mL}$, Sigma-Aldrich) for $12 \mathrm{~h}$ to mimic the activation of neutrophils in sepsis.

\section{Generation and differentiation of HL-60 cell lines with GSEC knocked down or overexpressed}

GSEC-knockdown and OE lentiviral vectors were constructed by GeneChem (Shanghai, China). Briefly, 2 target sequences (5'-GGTCACAACAG TACAAAGA-3' and 5'-CCAACTATGCCATGGTCTT-3') for GSEC were cloned into GV493 lentivirus vectors to construct knockdown lentiviral vectors of GSEC, and they were named lenti-GSEC-sh1 and lenti-GSEC-sh2 (Supplementary Fig. 22A and Supplementary Table 13), and a target sequence for GSEC (NONHSAT160878.1) was cloned into a GV367 lentiviral vector to construct OE lentiviral vectors of GSEC, named lenti-GSEC-OE (Supplementary Fig. 22B and Supplementary Table 13). Stable knockdown and $\mathrm{OE}$ cell lines were generated as previously described [51]. Briefly, HL-60 cells were transduced with lenti-GSEC-short hairpin RNA (shRNA) virus, lentiGSEC-OE virus, or control lentiviral vectors, and cultured in RPMI-1640 medium supplemented with polybrene $(5 \mu \mathrm{g} / \mathrm{mL})$. After $72 \mathrm{~h}$ of transduction, puromycin $(5 \mu \mathrm{g} / \mathrm{mL})$ was added to the culture medium for stable cell line selection. GSEC expression was determined by quantitative reversetranscription PCR (qRT-PCR). The results showed that GSEC expression was significantly reduced in the lenti-GSEC-shRNA-transfected cells (Supplementary Fig. 23A) and increased in the lenti-GSEC-OE-transfected cells (Supplementary Fig. 23B). The lentiviral vectors did not affect GSEC expression. We also found that the lentiviral vectors did not affect the expression of PFKFB3 mRNA in dHL-60 cells with or without LPS stimulation (Supplementary Fig. 24).

To induce the differentiation of neutrophil-like HL-60 (dHL-60) cells, HL60 cells were cultured in the presence of $1.25 \%$ DMSO for 6 days. The dHL60 cells were then collected and stimulated with LPS $(1 \mu \mathrm{g} / \mathrm{mL})$ for $12 \mathrm{~h}$.

\section{Quantitative reverse-transcription PCR}

qRT-PCR was performed to measure gene (GSEC, PFKFB3, TNF- $\alpha$, IL-1 $\beta$, and IL-6) expression as previously described [88]. Total RNA in neutrophils was extracted with TRIzol reagent (Qiagen) and a RNeasy kit (Qiagen) according to the manufacturer's instructions. RNA purity was determined by using a NanoDrop ND-1000 spectrophotometer (Thermo Fisher Scientific), and RNA integrity was evaluated by standard denaturing agarose gel electrophoresis. Then, RT-qPCR was performed using one step SYBR Premix Ex Taq (Roche) according to the manufacturer's protocol. After PCR amplification, the comparative $\mathrm{Ct}$ method was used for relative quantification of gene expression, which was normalized to $\beta$-actin expression. The following primers were used: GSEC sense primer $5^{\prime}$-GAGTTCATTTGCTCTCTCTGGCAC-3' and antisense primer $5^{\prime}$-AAGA GGAGGCCTGATGGGGATA-3'; PFKFB3 sense primer 5'-ATTGCGGTIT CGATGCCAC- $3^{\prime}$ and antisense primer $5^{\prime}$-GCCACAACTGTAGGGTCGT- $3^{\prime}$; TNF-a sense primer $5^{\prime}$-CCTCTCTCTAATCAGCCCTCTG-3' and antisense primer 5'-GAGGACCTGGGAGTAGATGAG-3'; IL-1 $\beta$ sense primer $5^{\prime}$-AGCT ACGAATCTCCGACCAC- $3^{\prime}$ and antisense primer $5^{\prime}$-CGTTATCCCATGTGT CGAAGAA-3'; and IL-6 sense primer 5'-CACAGACAGCCACTCACC- $3^{\prime}$ and antisense primer 5'-AGTGCCTCTTTGCTGCTTTC-3'.

\section{Dual-luciferase reporter assays}

Dual-luciferase reporter assays were used to demonstrate whether GSEC IncRNA can regulate the transcription of PFKFB3 in dHL-60 cells [89]. First, the PFKFB3 plasmids were constructed by GenePharma for use in dualluciferase reporter assays (Shanghai, China). Briefly, two wild-type PFKFB3 genes and corresponding mutants were cloned into PGL3B vectors to construct luciferase reporter PFKFB3 plasmids, named PFKFB3-wt1, PFKFB3-mut1, PFKFB3-wt2, and PFKFB3-mut2. Then, dHL-60 cells $(1.2 \times$ $10^{5}$ ) were grown in 96-well plates and co-transfected with the blank plasmids PFKFB3-wt 1/2 (with or without lenti-GSEC-OE) and PFKFB3-mut1/ 2 (with or without lenti-GSEC-OE). Firefly and Renilla luciferase activities were measured $48 \mathrm{~h}$ after transfection using a Dual-Glo Luciferase Assay
System (Promega, WI, USA) following the manufacturer's protocol. The firefly luciferase activity was normalized to the Renilla luciferase activity.

\section{PFKFB3 mRNA stability assay}

PFKFB3 mRNA stability was determined by qRT-PCR as previously described [88]. Briefly, GSEC expression was knocked down by infection with lenti-GSEC-shRNA. The cells were collected and stimulated with LPS $(1 \mu \mathrm{g} / \mathrm{mL})$ for $12 \mathrm{~h}$ to mimic activated neutrophils in sepsis. Then, actinomycin $D(5 \mu \mathrm{g} / \mathrm{mL})$ was added to the cell medium to block de novo RNA synthesis. Total RNA was collected at $0,1,2$, and $3 \mathrm{~h}$ following the addition of actinomycin D. PFKFB3 mRNA expression was measured by qRT-PCR. The PFKFB3 mRNA half-life was determined by comparing to the mRNA level before and after actinomycin D was added.

\section{Glycolysis assay}

The ECAR was measured using a Seahorse XF96 Flux Analyzer (Agilent) according to the manufacturer's instructions [90]. Briefly, cells were stimulated with LPS $(1 \mu \mathrm{g} / \mathrm{mL})$ for $12 \mathrm{~h}$ and then collected. For the ECAR determination, $1 \mu \mathrm{mol} / \mathrm{L}$ oligomycin, $0.5 \mu \mathrm{mol} / \mathrm{L}$ carbonyl cyanide $\mathrm{p}$ trifluoromethoxyphenylhydrazone, and $0.5 \mu \mathrm{mol} / \mathrm{L}$ rotenone plus $0.1 \mu \mathrm{mol} / \mathrm{L}$ antimycin A were injected into the wells. The ECAR were measured in 2 min intervals.

\section{Inflammatory cytokines}

The levels of inflammatory cytokines (TNF-a, IL-1 $\beta$, and IL-6) were determined by ELISA kits according to the manufacturer's instructions. Briefly, the supernatant of LPS $(1 \mu \mathrm{g} / \mathrm{mL}$, for $12 \mathrm{~h})$-stimulated $\mathrm{dHL}-60$ cells was added to a 96-well microtiter plate that contained a specific immobilized antibody that could bind TNF- $\alpha$, IL-1 $\beta$, or IL- 6 in the aliquot. After unbound substances and antibodies were removed with several washings and when color development was stopped by sulfuric acid, the optical density was determined at $540 \mathrm{~nm}$ in an ELISA plate reader. The results were calculated on a standard curve concentration and multiplied by the dilution factor.

\section{Western blot analysis}

Both neutrophils and dHL-60 cells were processed with the addition of RIPA buffer containing a protease inhibitor cocktail. For the detection of target proteins, the cell lysates were subjected to SDS electrophoresis on $10 \%$ polyacrylamide gels and transferred to nitrocellulose membranes. Following incubation with rabbit anti-PFKFB3 monoclonal antibody (CST) at $4{ }^{\circ} \mathrm{C}$ overnight, the membrane was incubated with the secondary antibody for $1 \mathrm{~h}$ at room temperature. Then, the band was visualized with an enhanced chemiluminescence (ECL) reagent and Hyper film ECL as described by the manufacturer.

\section{Statistical analysis}

Our data were statistically analyzed with GraphPad Prism software (version 8). Continuous data with a normal distribution are presented as the means \pm SD. Statistical analysis of two groups was performed with Student's $t$-tests. Categorical data are presented as number (\%). The statistical analysis of categorical data was performed with $x^{2}$-tests. Correlations were assessed by Pearson's analysis. An $a$-value of $P<0.05$ was considered statistically significant.

\section{DATA AVAILABILITY}

All datasets generated for this study are included in the article.

\section{REFERENCES}

1. Rhodes A, Evans LE, Alhazzani W, Levy MM, Antonelli M, Ferrer R, et al. Surviving sepsis campaign: international guidelines for management of sepsis and septic shock: 2016. Intensive Care Med. 2017;43:304-77.

2. Wentowski C, Mewada N, Nielsen ND. Sepsis in 2018: a review. Anaesth Intensive Care Med. 2019;20:6-13.

3. Suarez De La Rica A, Gilsanz F, Maseda E. Epidemiologic trends of sepsis in western countries. Ann Transl Med. 2016;4:325-31.

4. Cheng ML, Chien KY, Lai CH, Li GJ, Lin JF, Ho HY. Metabolic reprogramming of host cells in response to enteroviral infection. Cells. 2020;9:473-98.

5. Escoll P, Buchrieser C. Metabolic reprogramming of host cells upon bacterial infection: why shift to a Warburg-like metabolism? FEBS J. 2018;285:2146-60. 
6. Wyatt EV, Diaz K, Griffin AJ, Rasmussen JA, Crane DD, Jones BD, et al. Metabolic reprogramming of host cells by virulent Francisella tularensis for optimal replication and modulation of inflammation. J Immunol. 2016;196:4227-36.

7. Mantovani A, Cassatella MA, Costantini C, Jaillon S. Neutrophils in the activation and regulation of innate and adaptive immunity. Nat Rev Immunol. 2011;11:519-31.

8. Sonego F, Castanheira FV, Ferreira RG, Kanashiro A, Leite CA, Nascimento DC, et al. Paradoxical roles of the neutrophil in sepsis: protective and deleterious. Front Immunol. 2016;7:155-62.

9. Nathan C. Neutrophils and immunity: challenges and opportunities. Nat Rev Immunol. 2006;6:173-82.

10. Shen XF, Cao K, Jiang JP, Guan WX, Du JF. Neutrophil dysregulation during sepsis: an overview and update. J Cell Mol Med. 2017;21:1687-97.

11. Kumar S, Dikshit M. Metabolic insight of neutrophils in health and disease. Front Immunol. 2019:10:2099-116.

12. Li X, Kondo Y, Bao Y, Staudenmaier L, Lee A, Zhang J, et al. Systemic adenosine triphosphate impairs neutrophil chemotaxis and host defense in sepsis. Crit Care Med. 2017;45:e97-e104.

13. Kuwabara WM, Curi R, Alba-Loureiro TC. Autophagy is impaired in neutrophils from streptozotocin-induced diabetic rats. Front Immunol. 2017;8:24-34.

14. Drechsler $M$, Megens RT, van Zandvoort $M$, Weber $C$, Soehnlein $O$. Hyperlipidemia-triggered neutrophilia promotes early atherosclerosis. Circulation. 2010;122:1837-45.

15. Huang SC, Smith AM, Everts B, Colonna M, Pearce EL, Schilling JD, et al. Metabolic reprogramming mediated by the mTORC2-IRF4 signaling axis is essential for macrophage alternative activation. Immunity. 2016:45:817-30.

16. O'Neill LA, Kishton RJ, Rathmell J. A guide to immunometabolism for immunologists. Nat Rev Immunol. 2016;16:553-65.

17. Rodriguez-Espinosa O, Rojas-Espinosa O, Moreno-Altamirano MM, Lopez-Villegas EO, Sanchez-Garcia FJ. Metabolic requirements for neutrophil extracellular traps formation. Immunology. 2015;145:213-24.

18. Sadiku P, Willson JA, Dickinson RS, Murphy F, Harris AJ, Lewis A, et al. Prolyl hydroxylase 2 inactivation enhances glycogen storage and promotes excessive neutrophilic responses. J Clin Invest. 2017;127:3407-20.

19. Li JJ, Mao XH, Tian T, Wang WM, Su T, Jiang CH, et al. Role of PFKFB3 and CD163 in oral squamous cell carcinoma angiogenesis. Curr Med Sci. 2019;39:410-4.

20. De Bock K, Georgiadou M, Schoors S, Kuchnio A, Wong BW, Cantelmo AR, et al. Role of PFKFB3-driven glycolysis in vessel sprouting. Cell. 2013;154:651-63.

21. Zhang J, Zhang $\mathrm{Y}$, Wang J, Zhang $\mathrm{S}$, Zhao $\mathrm{Y}$, Ren $\mathrm{H}$, et al. Protein kinase D3 promotes gastric cancer development through p65/6-phosphofructo-2-kinase/ fructose-2,6-biphosphatase 3 activation of glycolysis. Exp Cell Res. 2019;380:188-97.

22. Wang J, Li X, Xiao Z, Wang Y, Han Y, Li J, et al. MicroRNA-488 inhibits proliferation and glycolysis in human prostate cancer cells by regulating PFKFB3. FEBS Open Biol. 2019;9:1798-807.

23. Cantelmo AR, Conradi LC, Brajic A, Goveia J, Kalucka J, Pircher A, et al. Inhibition of the glycolytic activator PFKFB3 in endothelium induces tumor vessel normalization, impairs metastasis, and improves chemotherapy. Cancer Cell. 2016;30:968-85.

24. Mercer TR, Dinger ME, Mattick JS. Long non-coding RNAs: insights into functions. Nat Rev Genet. 2009;10:155-9.

25. Geisler S, Coller J. RNA in unexpected places: long non-coding RNA functions in diverse cellular contexts. Nat Rev Mol Cell Biol. 2013;14:699-712.

26. Szczesniak MW, Makalowska I. IncRNA-RNA interactions across the human transcriptome. PLoS ONE. 2016;11:e0150353.

27. Li Y, Yang N, Zhou X, Bian X, Qiu G, Zhang M, et al. LncRNA and mRNA interaction study based on transcriptome profiles reveals potential core genes in the pathogenesis of human thoracic aortic dissection. Mol Med Rep. 2018;18:3167-76

28. Xing Z, Zhang Y, Liang K, Yan L, Xiang Y, Li C, et al. Expression of long noncoding RNA YIYA promotes glycolysis in breast cancer. Cancer Res. 2018;78:4524-32.

29. Chen F, Chen J, Yang L, Liu J, Zhang X, Zhang Y, et al. Extracellular vesiclepackaged HIF-1alpha-stabilizing IncRNA from tumour-associated macrophages regulates aerobic glycolysis of breast cancer cells. Nat Cell Biol. 2019;21:498-510.

30. Hua Q, Jin M, Mi B, Xu F, Li T, Zhao L, et al. LINC01123, a c-Myc-activated long non-coding RNA, promotes proliferation and aerobic glycolysis of non-small cell lung cancer through miR-199a-5p/c-Myc axis. J Hematol Oncol. 2019;12:91-109.

31. Reyes M, Filbin MR, Bhattacharyya RP, Billman K, Eisenhaure T, Hung DT, et al. An immune-cell signature of bacterial sepsis. Nat Med. 2020;26:333-40.

32. Wong HR, Cvijanovich N, Allen GL, Lin R, Anas N, Meyer K, et al. Genomic expression profiling across the pediatric systemic inflammatory response syndrome, sepsis, and septic shock spectrum. Crit Care Med. 2009;37:1558-66.

33. Sutherland A, Thomas M, Brandon RA, Brandon RB, Lipman J, Tang B, et al Development and validation of a novel molecular biomarker diagnostic test for the early detection of sepsis. Crit Care. 2011;15:R149.
34. Demaret J, Venet F, Friggeri A, Cazalis MA, Plassais J, Jallades L, et al. Marked alterations of neutrophil functions during sepsis-induced immunosuppression. J Leukoc Biol. 2015;98:1081-90.

35. Wong LL, Armugam A, Sepramaniam S, Karolina DS, Lim KY, Lim JY, et al. Circulating microRNAs in heart failure with reduced and preserved left ventricular ejection fraction. Eur J Heart Fail. 2015;17:393-404.

36. Wang SM, Liu GQ, Xian HB, Si JL, Qi SX, Yu YP. LncRNA NEAT1 alleviates sepsisinduced myocardial injury by regulating the TLR2/NF-kappaB signaling pathway. Eur Rev Med Pharm Sci. 2019;23:4898-907.

37. Birnie E, Weehuizen TAF, Lankelma JM, de Jong HK, Koh G, van Lieshout MHP, et al. Role of Toll-like receptor 5 (TLR5) in experimental melioidosis. Infect Immun. 2019;87:409-22.

38. Lu X, Xue L, Sun W, Ye J, Zhu Z, Mei H. Identification of key pathogenic genes of sepsis based on the Gene Expression Omnibus database. Mol Med Rep. 2018;17:3042-54.

39. Hardie DG. AMP-activated/SNF1 protein kinases: conserved guardians of cellular energy. Nat Rev Mol Cell Biol. 2007;8:774-85.

40. Ma J, Wei K, Liu J, Tang K, Zhang H, Zhu L, et al. Glycogen metabolism regulates macrophage-mediated acute inflammatory responses. Nat Commun. 2020;11:1769.

41. Tarnopolsky MA. Myopathies related to glycogen metabolism disorders. Neurotherapeutics. 2018;15:915-27.

42. Signal B, Gloss BS, Dinger ME. Computational approaches for functional prediction and characterisation of long noncoding RNAs. Trends Genet. 2016;32:620-37

43. Ashburner M, Ball CA, Blake JA, Botstein D, Butler H, Cherry JM, et al. Gene ontology: tool for the unification of biology. The Gene Ontology Consortium. Nat Genet. 2000;25:25-29.

44. Marko AJ, Miller RA, Kelman A, Frauwirth KA. Induction of glucose metabolism in stimulated $T$ lymphocytes is regulated by mitogen-activated protein kinase signaling. PLoS ONE. 2010;5:e15425.

45. Maciver NJ, Jacobs SR, Wieman HL, Wofford JA, Coloff JL, Rathmell JC. Glucose metabolism in lymphocytes is a regulated process with significant effects on immune cell function and survival. J Leukoc Biol. 2008;84:949-57.

46. Ardawi MS. Glutamine and glucose metabolism in human peripheral lymphocytes. Metabolism. 1988;37:99-103.

47. Wrzodek C, Drager A, Zell A. KEGGtranslator: visualizing and converting the KEGG PATHWAY database to various formats. Bioinformatics. 2011;27:2314-5.

48. Mondal S, Hsiao K, Goueli SA. Utility of adenosine monophosphate detection system for monitoring the activities of diverse enzyme reactions. Assay Drug Dev Technol. 2017;15:330-41.

49. Matsumura K, Kawasaki Y, Miyamoto M, Kamoshida Y, Nakamura J, Negishi L, et al. The novel G-quadruplex-containing long non-coding RNA GSEC antagonizes DHX36 and modulates colon cancer cell migration. Oncogene. 2017;36:1191-9.

50. Wang $X$, Qin W, Xu X, Xiong $Y$, Zhang $Y$, Zhang $H$, et al. Endotoxin-induced autocrine ATP signaling inhibits neutrophil chemotaxis through enhancing myosin light chain phosphorylation. Proc Natl Acad Sci USA. 2017;114:4483-8.

51. Liu X, Ma B, Malik AB, Tang H, Yang $T$, Sun B, et al. Bidirectional regulation of neutrophil migration by mitogen-activated protein kinases. Nat Immunol 2012;13:457-64.

52. Hombach S, Kretz M. Non-coding RNAs: classification, biology and functioning Adv Exp Med Biol. 2016;937:3-17.

53. Feng Y, Gao L, Cui G, Cao Y. LncRNA NEAT1 facilitates pancreatic cancer growth and metastasis through stabilizing ELF3 mRNA. Am J Cancer Res. 2020;10:237-48.

54. Angus DC, van der Poll T. Severe sepsis and septic shock. N Engl J Med. 2013;369:840-51.

55. Peters van Ton AM, Kox M, Abdo WF, Pickkers P. Precision immunotherapy for sepsis. Front Immunol. 2018;9:1926-36.

56. Kelly B, O'Neill LA. Metabolic reprogramming in macrophages and dendritic cells in innate immunity. Cell Res. 2015;25:771-84.

57. Weis S, Carlos AR, Moita MR, Singh S, Blankenhaus B, Cardoso S, et al. Metabolic adaptation establishes disease tolerance to sepsis. Cell. 2017;169:1263-75 e14.

58. Fitzpatrick SF, Immunometabolism, Sepsis. A role for HIF? Front Mol Biosci. 2019;6:85-99.

59. Cheng SC, Scicluna BP, Arts RJ, Gresnigt MS, Lachmandas E, GiamarellosBourboulis EJ, et al. Broad defects in the energy metabolism of leukocytes underlie immunoparalysis in sepsis. Nat Immunol. 2016;17:406-13.

60. Freemerman AJ, Johnson AR, Sacks GN, Milner JJ, Kirk EL, Troester MA, et al. Metabolic reprogramming of macrophages: glucose transporter 1 (GLUT1)mediated glucose metabolism drives a proinflammatory phenotype. J Biol Chem. 2014;289:7884-96.

61. Krawczyk CM, Holowka T, Sun J, Blagih J, Amiel E, DeBerardinis RJ, et al. Toll-like receptor-induced changes in glycolytic metabolism regulate dendritic cell activation. Blood. 2010;115:4742-9. 
62. Hotamisligil GS. Inflammation, metaflammation and immunometabolic disorders. Nature. 2017;542:177-85.

63. Curtis KD, Smith PR, Despres HW, Snyder JP, Hogan TC, Rodriguez PD, et al. Glycogen metabolism supports early glycolytic reprogramming and activation in dendritic in response to both TLR and Syk-dependent CLR agonists. Cells. 2020;9:715-25.

64. Everts B, Amiel E, Huang SC, Smith AM, Chang CH, Lam WY, et al. TLR-driven early glycolytic reprogramming via the kinases TBK1-IKKvarepsilon supports the anabolic demands of dendritic cell activation. Nat Immunol. 2014;15:323-32.

65. Van den Bossche J, O'Neill LA, Menon D. Macrophage immunometabolism: where are we (going)? Trends Immunol. 2017;38:395-406.

66. Yang $L$, Xie M, Yang M, Yu Y, Zhu S, Hou W, et al. PKM2 regulates the Warburg effect and promotes HMGB1 release in sepsis. Nat Commun. 2014;5:4436-55.

67. Haimovich B, Reddell MT, Calvano JE, Calvano SE, Macor MA, Coyle SM, et al. A novel model of common Toll-like receptor 4- and injury-induced transcriptional themes in human leukocytes. Crit Care. 2010;14:R177.

68. Robinson AJ, Hopkins GL, Rastogi N, Hodges M, Doyle M, Davies S, et al. Reactive oxygen species drive proliferation in acute myeloid leukemia via the glycolytic regulator PFKFB3. Cancer Res. 2020;80:937-49.

69. Lypova N, Telang S, Chesney J, Imbert-Fernandez Y. Increased 6-phosphofructo2-kinase/fructose-2,6-bisphosphatase-3 activity in response to EGFR signaling contributes to non-small cell lung cancer cell survival. J Biol Chem. 2019;294:10530-43.

70. Gu M, Li L, Zhang Z, Chen J, Zhang W, Zhang J, et al. PFKFB3 promotes proliferation, migration and angiogenesis in nasopharyngeal carcinoma. J Cancer. 2017:8:3887-96.

71. Jiang $H$, Shi $H$, Sun $M$, Wang $Y$, Meng $Q$, Guo $P$, et al. PFKFB3-driven macrophage glycolytic metabolism is a crucial component of innate antiviral defense. J Immunol. 2016;197:2880-90.

72. Zhang R, Li R, Liu Y, Li L, Tang Y. The glycolytic enzyme PFKFB3 controls TNFalpha-induced endothelial proinflammatory responses. Inflammation. 2019;42:146-55.

73. Wang Z, Kong L, Tan S, Zhang Y, Song X, Wang T, et al. Zhx2 accelerates sepsis by promoting macrophage glycolysis via Pfkfb3. J Immunol. 2020;204:2232-41.

74. Gong Y, Lan H, Yu Z, Wang M, Wang S, Chen Y, et al. Blockage of glycolysis by targeting PFKFB3 alleviates sepsis-related acute lung injury via suppressing inflammation and apoptosis of alveolar epithelial cells. Biochem Biophys Res Commun. 2017;491:522-9.

75. Wang L, Cao Y, Gorshkov B, Zhou Y, Yang Q, Xu J, et al. Ablation of endothelial $\mathrm{Pfkfb} 3$ protects mice from acute lung injury in LPS-induced endotoxemia. Pharm Res. 2019;146:104292-328.

76. Shankaraiah RC, Veronese A, Sabbioni S, Negrini M. Non-coding RNAs in the reprogramming of glucose metabolism in cancer. Cancer Lett. 2018;419:167-74.

77. Wang Y, Lu JH, Wu QN, Jin Y, Wang DS, Chen YX, et al. LncRNA LINRIS stabilizes IGF2BP2 and promotes the aerobic glycolysis in colorectal cancer. Mol Cancer. 2019;18:174-92.

78. Wang L, Cao L, Wen C, Li J, Yu G, Liu C. LncRNA LINC00857 regulates lung adenocarcinoma progression, apoptosis and glycolysis by targeting miR-1179/ SPAG5 axis. Hum Cell. 2020;33:195-204.

79. Carpenter S, Aiello D, Atianand MK, Ricci EP, Gandhi P, Hall LL, et al. A long noncoding RNA mediates both activation and repression of immune response genes. Science. 2013;341:789-92.

80. Liu J, Liu ZX, Wu QN, Lu YX, Wong CW, Miao L, et al. Long noncoding RNA AGPG regulates $\mathrm{PFKFB} 3-$ mediated tumor glycolytic reprogramming. Nat Commun. 2020;11:1507-23.

81. Gautier L, Cope L, Bolstad BM, Irizarry RA. affy-analysis of Affymetrix GeneChip data at the probe level. Bioinformatics. 2004;20:307-15.

82. Langfelder $P$, Horvath S. WGCNA: an R package for weighted correlation network analysis. BMC Bioinformatics. 2008;9:559-72.

83. Zhang B, Kirov S, Snoddy J. WebGestalt: an integrated system for exploring gene sets in various biological contexts. Nucleic Acids Res. 2005;33: W741-W748.

84. Huang DW, Sherman BT, Tan Q, Collins JR, Alvord WG, Roayaei J, et al. The DAVID Gene Functional Classification Tool: a novel biological module-centric algorithm to functionally analyze large gene lists. Genome Biol. 2007;8:R183.

85. Kuhns DB, Priel DAL, Chu J, Zarember KA. Isolation and functional analysis of human neutrophils. Curr Protoc Immunol. 2015;111:7231-72316.

86. Floyd H, Ni J, Cornish AL, Zeng Z, Liu D, Carter KC, et al. Siglec-8. A novel eosinophil-specific member of the immunoglobulin superfamily. J Biol Chem. 2000;275:861-6.

87. Hamdani SH, McMillan DN, Pettersen EF, Wergeland H, Endresen C, Ellis AE, et al. Isolation of rainbow trout neutrophils with an anti-granulocyte monoclonal antibody. Vet Immunol Immunopathol. 1998;63:369-80.
88. Song $H, X u Y$, Shi $L$, Xu T, Fan R, Cao M, et al. LncRNA THOR increases the stemness of gastric cancer cells via enhancing SOX9 mRNA stability. Biomed Pharmacother. 2018;108:338-46.

89. Wang X, Hu LP, Qin WT, Yang Q, Chen DY, Li Q, et al. Identification of a subset of immunosuppressive P2RX1-negative neutrophils in pancreatic cancer liver metastasis. Nat Commun. 2021;12:174-86.

90. Kenwood BM, Weaver JL, Bajwa A, Poon IK, Byrne FL, Murrow BA, et al. Identification of a novel mitochondrial uncoupler that does not depolarize the plasma membrane. Mol Metab. 2014;3:114-23.

\section{ACKNOWLEDGEMENTS}

We thank all of the patients and healthy volunteers who participated in this study for their contribution of blood specimens.

\section{AUTHOR CONTRIBUTIONS}

DDL, JTY, and KZ designed this study. DDL and WS performed the experiments. DYZ and JTY contributed to the collection of human patient samples and clinical data. DYZ, ZYY, and WTQ contributed to the data analysis. KZ and JTY provided suggestions for manuscript preparation. JTY contributed to the overall study design, data analysis, and manuscript preparation.

\section{FUNDING}

This study was supported by funds from the Zhenjiang Social Development Program (SH2020057) to JY and Jurong People's Livelihood Science and Technology Project (2020SA00105) to WS.

\section{ETHICS STATEMENT}

This study was approved by the Medical Ethical Committee of Jiangsu University. Blood specimens were obtained from the cubital veins of septic patients and healthy drug-free donors after receiving written informed consent. All of the experiments were performed in accordance with the approval guidelines.

\section{COMPETING INTERESTS}

The authors declare no competing interests.

\section{ADDITIONAL INFORMATION}

Supplementary information The online version contains supplementary material available at https://doi.org/10.1038/s41419-021-04428-7.

Correspondence and requests for materials should be addressed to Kai Zhang or Jiangtao Yin.

Reprints and permission information is available at http://www.nature.com/ reprints

Publisher's note Springer Nature remains neutral with regard to jurisdictional claims in published maps and institutional affiliations.

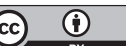

Open Access This article is licensed under a Creative Commons Attribution 4.0 International License, which permits use, sharing, adaptation, distribution and reproduction in any medium or format, as long as you give appropriate credit to the original author(s) and the source, provide a link to the Creative Commons license, and indicate if changes were made. The images or other third party material in this article are included in the article's Creative Commons license, unless indicated otherwise in a credit line to the material. If material is not included in the article's Creative Commons license and your intended use is not permitted by statutory regulation or exceeds the permitted use, you will need to obtain permission directly from the copyright holder. To view a copy of this license, visit http://creativecommons. org/licenses/by/4.0/.

(c) The Author(s) 2021 